\title{
Dark sectors with dynamical coupling
}

\author{
Weiqiang Yang, ${ }^{1, *}$ Olga Mena,$^{2, \uparrow}$ Supriya Pan $\odot,{ }^{3, *}$ and Eleonora Di Valentino, ${ }^{4, \S}$ \\ ${ }^{1}$ Department of Physics, Liaoning Normal University, Dalian 116029, People's Republic of China \\ ${ }^{2}$ IFIC, Universidad de Valencia-CSIC, 46071 Valencia, Spain \\ ${ }^{3}$ Department of Mathematics, Presidency University, 86/1 College Street, Kolkata 700073, India \\ ${ }^{4}$ Jodrell Bank Center for Astrophysics, School of Physics and Astronomy, University of Manchester, \\ Oxford Road, Manchester M13 9PL, United Kingdom
}

(Received 30 June 2019; published 7 October 2019)

\begin{abstract}
Coupled dark matter-dark energy scenarios are modeled via a dimensionless parameter $\xi$, which controls the strength of their interaction. While this coupling is commonly assumed to be constant, there is no underlying physical law or symmetry that forbids a time-dependent $\xi$ parameter. The most general and complete interacting scenarios between the two dark sectors should therefore allow for such a possibility, and it is the main purpose of this study to constrain two possible and well-motivated coupled cosmologies by means of the most recent and accurate early- and late-time universe observations. We find that $\mathrm{CMB}$ data alone prefer $\xi(z)>0$ and therefore a smaller amount of dark matter, alleviating some crucial and well-known cosmological data tensions. An objective assessment of the Bayesian evidence for the coupled models explored here shows no particular preference for the presence of a dynamical dark sector coupling.
\end{abstract}

DOI: 10.1103/PhysRevD.100.083509

\section{INTRODUCTION}

Dark matter and dark energy, according to a series of observational evidences, are the two main constituents of the Universe, comprising nearly $96 \%$ of its total energy density [1]. For the last 20 years, a huge observational effort has been devoted to unraveling the nature of these two fluids [2-6]. Despite the fact that some of their properties have been measured with unprecedented accuracy [the value of the dark energy equation of state with $95 \%$ CL errors is $w=-1.028_{-0.061}^{+0.063}$ from the latest Cosmic Microwave Background (CMB) data, combined with large scale structure observations and Supernovae Ia luminosity distances [1], their nature still remains obscure. Furthermore, the socalled "why now" problem provides another puzzle that may suggest a contemporary evolution of the two dark fluids. From the particle physics perspective, if a cosmic scalar field is responsible for the dark energy component, it may couple to all other fields in nature, if it is present [7]. These models emerged as coupled quintessence [8-13]. Indeed, the presence of an interaction between the two dark fluids could successfully address the cosmic coincidence problem. Furthermore, some quintessence models could also be interpreted as modified gravity (Brans-Dicke-like) theories. An extra bonus supporting interactions among the two dark

\footnotetext{
d11102004@163.com

omena@ific.uv.es

*supriya.maths@presiuniv.ac.in

§eleonora.divalentino@manchester.ac.uk
}

sectors arises from the fact that, when dark matter and dark energy interact, an effective equation of state $w<-1$ could naturally appear [14-16]. While plenty of work in the literature has been devoted to exploring the rich phenomenology of these models [17-43], more recently, an extra encouraging aspect of these theories has improved their role as an alternative to a pure $\Lambda$ CDM universe. Namely, in dark matter-dark energy coupled cosmologies, the tension between local and CMB estimations of the Hubble constant $H_{0}$ could be ameliorated [44-47].

Current cosmological observations still allow for significant interactions among the two dark sectors, i.e., between dark matter and dark energy; see e.g., Refs. [48-53]. In this work we consider an interacting scenario in which vacuum interacts with pressureless dark matter, adopting the more general phenomenological viewpoint, i.e., inspecting a timedependent coupling. Such a consideration also entails the case of a coupling parameter that remains constant in cosmic time. For our analyses we have assumed that our Universe is homogeneous and isotropic; that is, its geometry is well described by the Friedmann-Lemaitre-Robertson-Walker (FLRW) line element.

The work has been organized as follows. Section II contains the gravitational equations within an interacting universe. In Sec. III we describe the observational data and the methodology used to constrain the interacting dark energy models. Section IV presents the observational constraints on the models, including also a Bayesian evidence analysis. Finally, we draw our conclusions in Sec. V. 


\section{GRAVITATIONAL EQUATIONS OF A UNIVERSE WITH INTERACTING DARK SECTORS}

A homogeneous and isotropic universe is well described by the FLRW metric:

$d s^{2}=-d t^{2}+a^{2}(t)\left[\frac{d r^{2}}{1-\kappa r^{2}}+r^{2}\left(d \theta^{2}+\sin ^{2} \theta d \phi^{2}\right)\right]$,

in which $a(t)$ is the expansion scale factor of the universe and $\kappa=0,+1$, and -1 correspond to a spatially flat, closed, and open universe, respectively. In the following, we assume that the gravitational sector of the theory is described by the Einstein gravity and $\kappa=0$. Within this simple cosmological scenario, we introduce an interaction between the pressureless cold dark matter component and the dark energy fluid, acting as vacuum energy. All in all, the conservation equations read as

$$
\nabla^{\mu}\left(T_{\mu \nu}^{\mathrm{CDM}}+T_{\mu \nu}^{\mathrm{DE}}\right)=0,
$$

where $T_{\mu \nu}^{i}(i=\mathrm{CDM}, \mathrm{DE})$ is the energy-momentum tensor for the $i$ th dark sector. Considering the dark energy fluid as a cosmological constant with an equation of state $w_{x}=p_{x} / \rho_{x}=-1$, the conservation equations are given by

$$
\begin{gathered}
\dot{\rho}_{x}=Q, \\
\dot{\rho}_{c}=-3 H \rho_{c}-Q,
\end{gathered}
$$

where the dot refers to derivatives with respect to the time $t$, $H \equiv \dot{a} / a$ is the Hubble parameter, $\rho_{c}$ is the cold dark matter mass-energy density, and $Q$ encodes the interaction rate between the dark fluids. Our analyses will be applied to two possible models, named the interacting vacuum scenarios 1 and 2 (IVS1 and IVS2, respectively):

$$
\begin{gathered}
\text { IVS1: } Q=3 \xi(a) H \rho_{x}, \\
\text { IVS2: } Q=3 \xi(a) H \frac{\rho_{c} \rho_{x}}{\rho_{c}+\rho_{x}},
\end{gathered}
$$

where $\xi(a)$ is a time-dependent dimensionless coupling. A Taylor expansion of $\xi(a)$ around the present time $(a=1)$ leads to

$$
\xi(a)=\xi_{0}+(a-1) \xi^{\prime}\left(a_{0}=1\right)+\frac{(a-1)^{2}}{2 !} \xi^{\prime \prime}\left(a_{0}=1\right)
$$$$
+\cdots
$$

where the prime stands for the derivative with respect to the scale factor. In this work, we restrict ourselves to linear corrections of $\xi(a)$. We therefore consider the following parametrization of the time-dependent coupling parameter

$$
\xi(a)=\xi_{0}+\xi_{a}(1-a),
$$

where we use $\xi_{a}$ instead of $\xi^{\prime}\left(a_{0}=1\right)$. We note that the above choice was used recently by the authors of [54] and earlier in $[55,56]$.

For the first interaction model (IVS1) it is possible to obtain an analytical solution for the background evolution of the dark sector fluids:

$$
\begin{gathered}
\rho_{x}=\rho_{x 0} a^{3\left(\xi_{0}+\xi_{a}\right)} \exp \left(-3 \xi_{a}(a-1)\right), \\
\rho_{c}=\rho_{c 0} a^{-3}-3 \rho_{x 0} a^{-3} \int_{1}^{a}\left[\xi_{0}+\xi_{a}(1-a)\right] a^{3\left(\xi_{0}+\xi_{a}\right)+2} \\
\times \exp \left(-3 \xi_{a}(a-1)\right) .
\end{gathered}
$$

For the IVS2 model the background evolution needs to be computed numerically.

To evaluate the perturbation equations in the presence of an interaction, we work within the perturbed FLRW metric [57-59] and follow the synchronous gauge; see Ref. [60] for details.

Last but not least, we note that if in IVS1 the interaction function is simply considered as $Q=\Gamma \rho_{x}$, where $\Gamma$ is purely a time-independent constant, that means if the interaction rate depends solely on the dark energy component not on other physical parameters - such as the Hubble expansion rate, scale factor of the universe, etc., it is then believed that such cosmological models should reflect more light on the intrinsic properties of the dark energy. Similarly, without assuming any interaction in the dark sector, one could also construct some physically motivated phenomenological dark energy models where dark energy could decay with the evolution of the universe, leading to a class of metastable dark energy models [61-64].

\section{OBSERVATIONAL DATA AND STATISTICAL METHOD}

In this section we describe the cosmological observations that we have used to constrain the interacting scenarios. A discussion concerning the statistical method used in our analyses is also detailed. The publicly available data sets that we exploit in this paper are as follows:

(i) Cosmic microwave background $(C M B)$.- We use the cosmic microwave background measurements from the Planck 2015 data release [65,66], which includes both the high- $\ell(30 \leq \ell \leq 2508)$ TT and the low- $\ell(2 \leq \ell \leq 29)$ TT likelihoods. The Planck polarization likelihood in the low- $\ell$ multipole regime $(2 \leq \ell \leq 29)$, together with the high-multipole $(30 \leq \ell \leq 1996) \mathrm{EE}$ and TE likelihoods, is also considered. Despite the fact that all these likelihoods have a clear dependence on a given number of nuisance parameters, such as residual foreground contamination, calibration, and others, we have also 
TABLE I. Flat priors imposed on various cosmological parameters of the interacting dark energy scenarios.

\begin{tabular}{lc}
\hline \hline Parameter & Prior \\
\hline$\Omega_{b} h^{2}$ & {$[0.005,0.1]$} \\
$\Omega_{c} h^{2}$ & {$[0.01,0.99]$} \\
$\tau$ & {$[0.01,0.8]$} \\
$n_{s}$ & {$[0.5,1.5]$} \\
$\log \left[10^{10} A_{S}\right]$ & {$[2.4,4]$} \\
$100 \theta_{\mathrm{MC}}$ & {$[0.5,10]$} \\
$\xi_{0}$ & {$[-1,1]$} \\
$\xi_{a}$ & {$[-1,1]$} \\
\hline \hline
\end{tabular}

accounted for those in our numerical analyses and marginalized over them when presenting the final constraints.

(ii) Baryon acoustic oscillation (BAO) distance measurements.-We use the BAO data from different observational missions; see Refs. [67-69].

(iii) Supernovae type Ia (Pantheon).- -The supernovae type Ia (SNIa) were the first indicators for an accelerating phase of the universe. Here, we use the latest compilation of SNIa data (known as the Pantheon sample) comprising 1048 data points [70].

(iv) Hubble constant (R19).--Finally, we also consider the impact of a recent estimation of the Hubble constant, $H_{0}=74.03 \pm 1.42 \mathrm{~km} / \mathrm{s} / \mathrm{Mpc}$ at $68 \% \mathrm{CL}$

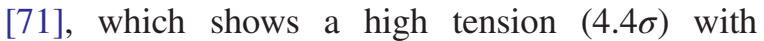
$\mathrm{CMB}$ estimates within the minimal $\Lambda \mathrm{CDM}$ cosmological model.

However, we combine these data sets in the context of our IVS1 and IVS2 dark matter-dark energy models [see Eqs. (5) and (6)] to explore whether this tension could be alleviated within these nonstandard cosmologies.

For the statistical analyses, we make use of cosmomc, a Markov chain Monte Carlo package [72,73], equipped with the Gelman and Rubin statistics for convergence diagnosis. This software also includes the support for the Planck 2015 likelihood [66]. The parameter space we constrain is

$$
\mathcal{P} \equiv\left\{\Omega_{b} h^{2}, \Omega_{c} h^{2}, 100 \theta_{\mathrm{MC}}, \tau, \xi_{0}, \xi_{a}, n_{s}, \log \left[10^{10} A_{S}\right]\right\},
$$

in which $\Omega_{b} h^{2}$ is the physical density for baryons; $\Omega_{c} h^{2}$ is the physical density for $\mathrm{CDM} ; \theta_{\mathrm{MC}}$ denotes the ratio of the sound horizon to the angular diameter distance; $\tau$ is the reionization optical depth; $n_{s}$ denotes the scalar spectral index; $A_{S}$ denotes the amplitude of the primordial scalar power spectrum; and $\xi_{0}, \xi_{a}$ control the interaction rate among the two dark sectors. We are therefore exploring an eight-dimensional parameter space with two extra degrees of freedom compared to the six-dimensional $\Lambda \mathrm{CDM}$ cosmology. We note that in the present work we are considering the spatially flat FLRW universe. Table I presents the priors imposed on the model parameters for the statistical analyses.

\section{NUMERICAL ANALYSES AND RESULTS}

In this section we present the constraints on the interacting scenarios IVS1 and IVS2 [Eqs. (5) and (6), respectively], arising from the combination of several data sets, namely, $\mathrm{CMB}, \mathrm{CMB}+\mathrm{R} 19, \mathrm{CMB}+\mathrm{BAO}, \mathrm{CMB}+$ $\mathrm{BAO}+\mathrm{R} 19$, and $\mathrm{CMB}+\mathrm{BAO}+$ Pantheon.

$$
\text { A. IVS1: } Q=3\left[\xi_{0}+\xi_{a}(1-a)\right] H \rho_{x}
$$

The observational constraints for this interacting dark energy scenario have been displayed in Table II. Figures 1 and 2 depict the one-dimensional marginalized posterior distributions and the two-dimensional joint contours for some selected cosmological parameters. Notice from Table II that the mean values of the parameters $\xi_{0}$ and $\xi_{a}$, quantifying the interaction among the dark sectors, are

TABLE II. Mean values and $95 \% \mathrm{CL}$ errors on the cosmological parameters for the IVS1 interacting scenario,

\begin{tabular}{|c|c|c|c|c|c|}
\hline Parameters & CMB & $\mathrm{CMB}+\mathrm{BAO}$ & $\mathrm{CMB}+\mathrm{BAO}+$ Pantheon & $\mathrm{CMB}+\mathrm{R} 19$ & $\mathrm{CMB}+\mathrm{BAO}+\mathrm{R} 19$ \\
\hline$\Omega_{c} h^{2}$ & $0.106_{-0.040}^{+0.039}$ & $0.116_{-0.044}^{0.035}$ & $0.121_{-0.023}^{+0.025}$ & $0.084_{-0.026}^{+0.032}$ & $0.085_{-0.030}^{+0.037}$ \\
\hline$\Omega_{b} h^{2}$ & $0.02220_{-0.00031}^{+0.00032}$ & $0.02224_{-0.00029}^{+0.00031}$ & $0.02226_{-0.00031}^{+0.00029}$ & $0.02223_{-0.00028}^{+0.00031}$ & $0.02223_{-0}^{+0.000039}$ \\
\hline $100 \theta_{\mathrm{MC}}$ & $1.0413_{-0.0025}^{+0.0027}$ & $1.0408_{-0.0020}^{+0.0027}$ & $1.0404_{-0.0015}^{+0.0014}$ & $1.0426_{-0.0021}^{1+0.0018}$ & $1.0426_{-0.0023}^{1+0.0019}$ \\
\hline$\tau$ & $0.079_{-0.033}^{+0.035}$ & $0.082_{-0.036}^{+0.035}$ & $0.083_{-0.036}^{+0.036}$ & $0.077_{-0.032}^{+0.045}$ & $0.078_{-0.036}^{+0.035}$ \\
\hline$n_{s}$ & $0.9731_{-0.0084}^{+0.0088}$ & $0.9747_{-0.0084}^{+0.0081}$ & $0.9750_{-0.0084}^{+0.0085}$ & $0.9740_{-0.0082}^{+0.0086}$ & $0.9741_{-0.0090}^{+0.0090}$ \\
\hline $\ln \left(10^{10} A_{S}\right)$ & $3.101_{-0.065}^{+0.066}$ & $3.106_{-0.070}^{+0.068}$ & $3.108_{-0.071}^{+0.071}$ & $3.096_{-0.062}^{+0.0652}$ & $3.100_{-0.069}^{+0.069}$ \\
\hline$\xi_{0}$ & $-0.01_{-0.21}^{+0.21}$ & $-0.04_{-0.20}^{+0.23}$ & $-0.04_{-0.17}^{+0.15}$ & $-0.03_{-0.17}^{+0.16}$ & $0.05_{-0.21}^{+0.18}$ \\
\hline$\xi_{a}$ & $0.20_{-0.56}^{+0.71}$ & $0.18_{-0.46}^{+0.50}$ & $0.14_{-037}^{+0.45}$ & $0.55_{-0.52}^{+0.50}$ & $0.19_{-0.48}^{+0.52}$ \\
\hline$\Omega_{m 0}$ & $0.27_{-0.12}^{+0.13}$ & $0.30_{-0.11}^{+0.09}$ & $0.313_{-0.058}^{+0.062}$ & $0.202_{-0.054}^{+0.067}$ & $0.214_{-0.066}^{+0.082}$ \\
\hline$\sigma_{8}$ & $0.94_{-0.28}^{+0.12}$ & $0.87_{-0.22}^{+0.34}$ & $0.82_{-0.14}^{+0.030}$ & $1.09_{-0.27}^{+0.034}$ & $1.10_{-0.32}^{+0.080}$ \\
\hline$H_{0}$ & $69.2_{-5.0}^{+5.0}$ & $68.5_{-2.4}^{+2.6}$ & $68.0_{-1.6}^{+1.6}$ & $72.9_{-2.4}^{+2.3}$ & $71.1_{-1.9}^{+2.3}$ \\
\hline$S_{8}$ & $0.86_{-0.09}^{+0.13}$ & $0.84_{-0.07}^{+0.14}$ & $0.827_{-0.060}^{+0.065}$ & $0.89_{-0.11}^{+0.10}$ & $0.91_{-0.11}^{+0.09}$ \\
\hline
\end{tabular}
$Q=3\left[\xi_{0}+\xi_{a}(1-a)\right] H \rho_{x}$, using different combinations of the cosmological data sets considered here. 


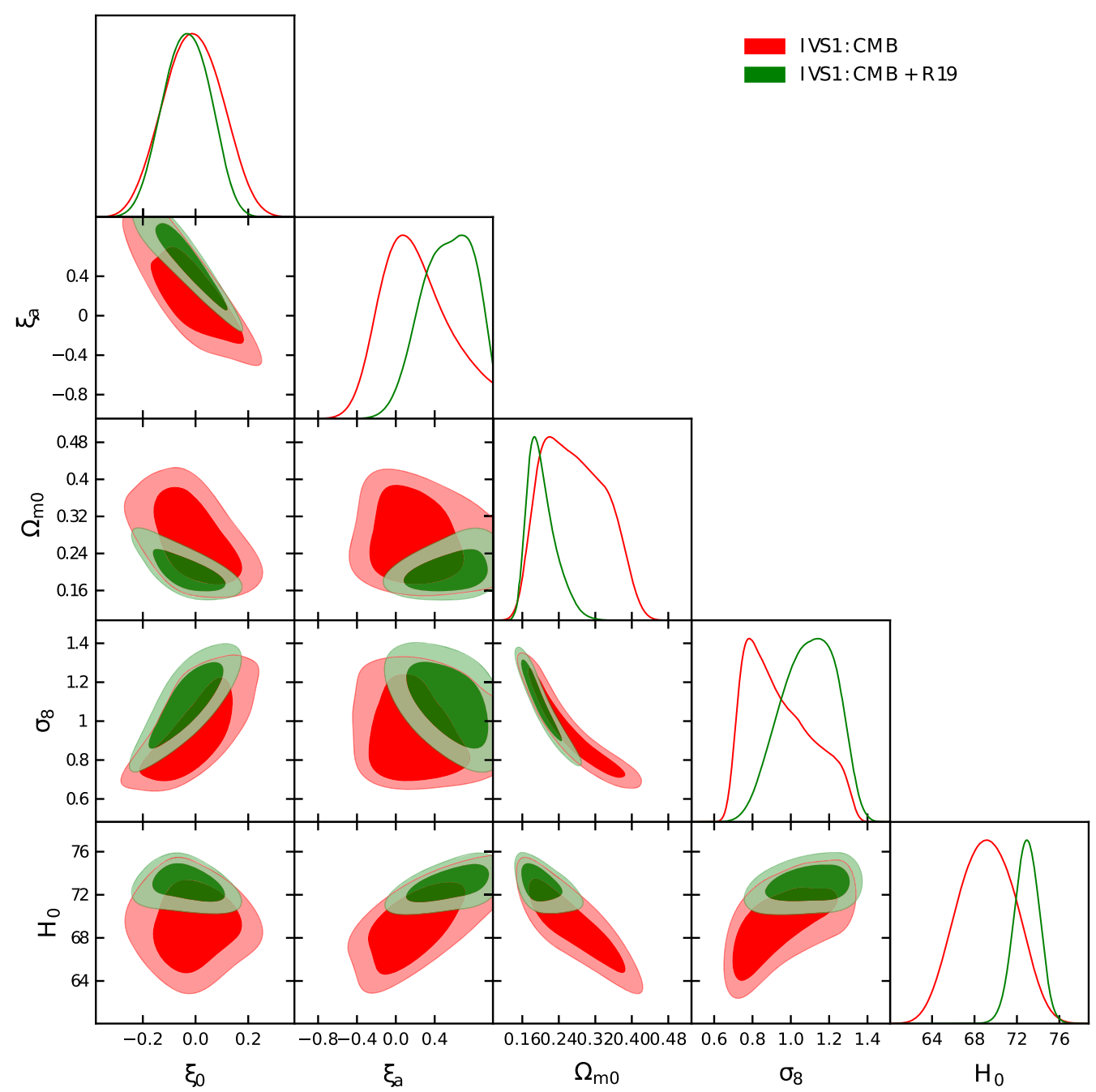

FIG. 1. Two-dimensional contours and one-dimensional marginalized posterior distributions for some key parameters of the IVS1 scenario for the $\mathrm{CMB}$ and $\mathrm{CMB}+\mathrm{R} 19$ data sets.

of opposite signs, and even if values $\left(\xi_{0}, \xi_{a}\right) \neq(0,0)$ are still allowed by the observational data, a noninteracting scenario is consistent within $68 \% \mathrm{CL}$. Figures 1 and 2 show the strong anticorrelation between the interaction parameters $\xi_{0}$ and $\xi_{a}$.

Concerning the value of $H_{0}$ within the IVS1 interacting scheme, note that it is slightly larger than that obtained with Planck CMB data alone in the context of a $\Lambda$ CDM model [6]. Due to the larger error bars on $H_{0}$, the $4 \sigma$ tension between local measurements $(74.03 \pm 1.42 \mathrm{~km} / \mathrm{s} / \mathrm{Mpc}$ [71]) and $\mathrm{CMB}$ observations is reduced to 2 standard deviations. Combining the CMB data set with a Gaussian prior on $H_{0}$ from R19 (see the fifth column of Table II), we obtain $\xi_{a}=0.55_{-0.52}^{+0.50}$ at $95 \%$ CL, i.e., different from zero with a significance of 2 standard deviations. The dynamical evolution of the coupling parameter $\xi(z)$ is illustrated on the left panel of Fig. 3, considering the CMB (solid curve) and the $\mathrm{CMB}+\mathrm{R} 19$ data combination (dotted curve), where all the parameters have been fixed to their mean values.
As for the $\mathrm{CMB}+\mathrm{R} 19$ data combination, the dynamical coupling $\xi(z)$ is larger than zero, the matter density shifts towards a lower value (see the strong anticorrelation between $\Omega_{m 0}$ and $\xi_{0}$ in Fig. 1), and therefore there is not an increase in the quantity $S_{8} \equiv \sigma_{8} \sqrt{\Omega_{m 0} / 0.3}$. Consequently, in this case, the tension at more than $2 \sigma$ on $S_{8}$ [74] between Planck and the cosmic shear experiments, namely, KiDS-450 [75-77], DES [78,79], and CFHTLenS [80-82], is solved within 1 standard deviation.

When adding the BAO data set, the error bars on the Hubble constant are notably decreased with respect to what we observed with the CMB alone. ${ }^{1}$ In this case the tension with R19 is only mildly alleviated, as it remains present at the $3 \sigma$ level. The addition of BAO measurements brings the dynamical evolution of the dark sector coupling very close to zero; see the right panel of Fig. 3.

\footnotetext{
${ }^{1}$ The inclusion of BAO data in other data sets has a notable effect on the error bars on $H_{0}$; see for instance $[42,83,84]$.
} 


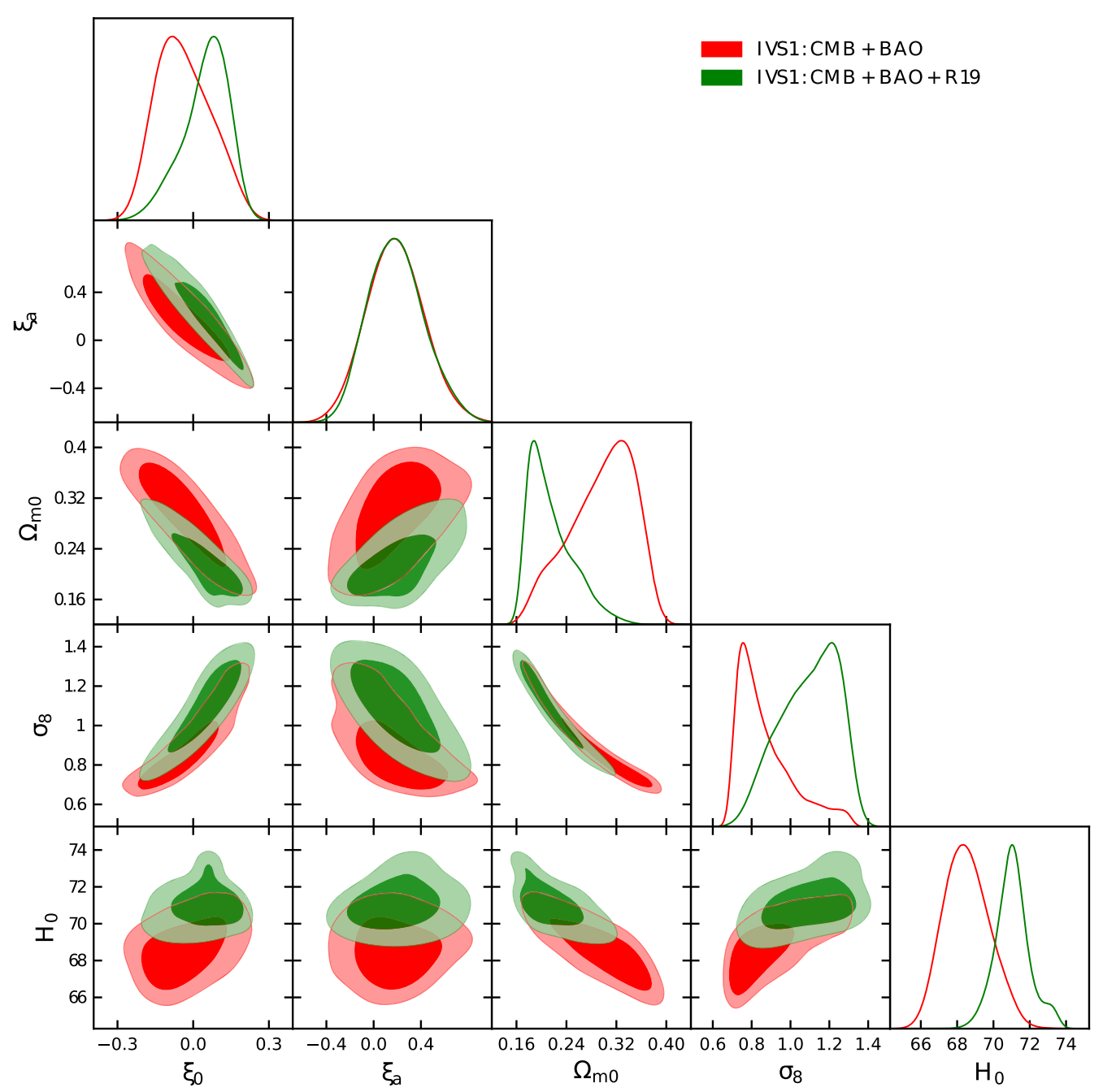

FIG. 2. Two-dimensional contours and one-dimensional marginalized posterior distributions for some key parameters of the IVS1 scenario for the $\mathrm{CMB}+\mathrm{BAO}$ and $\mathrm{CMB}+\mathrm{BAO}+\mathrm{R} 19$ data sets.
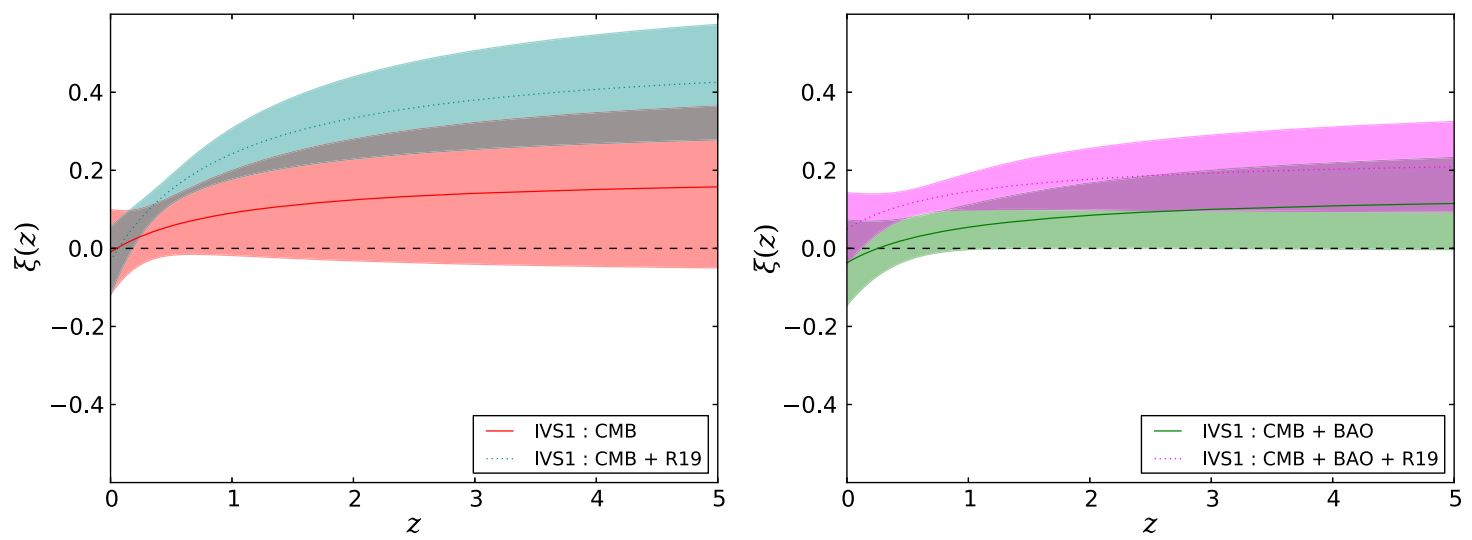

FIG. 3. Redshift evolution of the dynamical coupling parameter $\xi(z)\left(1+z=a^{-1}\right)$ for the IVS1 scenario using various observational data sets. The left panel corresponds to the $\mathrm{CMB}$ and $\mathrm{CMB}+\mathrm{R} 19$ data sets, while the right panel stands for the $\mathrm{CMB}+\mathrm{BAO}$ and $\mathrm{CMB}+\mathrm{BAO}+\mathrm{R} 19$ data sets. 
TABLE III. Mean values and 95\% CL errors on the cosmological parameters of the IVS2 interacting scenario, $Q=3\left[\xi_{0}+\xi_{a}(1-a)\right] H \frac{\rho_{c} \rho_{x}}{\rho_{c}+\rho_{x}}$, using different combinations of the cosmological data sets considered here.

\begin{tabular}{|c|c|c|c|c|c|}
\hline Parameters & $\mathrm{CMB}$ & $\mathrm{CMB}+\mathrm{BAO}$ & $\mathrm{CMB}+\mathrm{BAO}+$ Pantheon & $\mathrm{CMB}+\mathrm{R} 19$ & $\mathrm{CMB}+\mathrm{BA} 0+\mathrm{R} 19$ \\
\hline$\Omega_{c} h^{2}$ & $0.114_{-0.048}^{+0.043}$ & $0.125_{-0.036}^{+0.031}$ & $0.120_{-0.032}^{+0.031}$ & $0.081_{-0.019}^{+0.022}$ & $0.093_{-0.022}^{+0.026}$ \\
\hline$\Omega_{b} h^{2}$ & $0.02219_{-0.00030}^{+0.00031}$ & $0.02225_{-0.00030}^{+0.000029}$ & $0.02226_{-0.00030}^{+0.000030}$ & $0.02223_{-0.00028}^{+0.000030}$ & $0.02220_{-0.00030}^{+0.00028}$ \\
\hline $100 \theta_{\mathrm{MC}}$ & $1.0408_{-0.0025}^{+0.0027}$ & $1.0402_{-0.0018}^{+0.0000}$ & $1.0405_{-0.0016}^{+0.0017}$ & $1.0428_{-0.0020}^{+0.0013}$ & $1.0420_{-0.0016}^{+0.0015}$ \\
\hline$\tau$ & $0.080_{-0.034}^{+0.034}$ & $0.083_{-0.034}^{+0.034}$ & $0.084_{-0.034}^{+0.033}$ & $0.086_{-0.032}^{+0.030}$ & $0.080_{-0.037}^{+0.037}$ \\
\hline$n_{s}$ & $0.9733_{-0.0085}^{+0.0095}$ & $0.9749_{-0.0088}^{+0.0087}$ & $0.9755_{-0.0078}^{+0.0081}$ & $0.9751_{-0.0082}^{+0.0081}$ & $0.972_{-0.010}^{+0.011}$ \\
\hline $\ln \left(10^{10} A_{S}\right)$ & $3.105_{-0.067}^{+0.066}$ & $3.108_{-0.066}^{+0.066}$ & $3.109_{-0.066}^{+0.064}$ & $3.114_{-0.067}^{+0.066}$ & $3.106_{-0.072}^{+0.073}$ \\
\hline$\xi_{0}$ & $-0.02_{-0.38}^{+0.43}$ & $-0.17_{-0.38}^{+0.41}$ & $-0.06_{-0.40}^{+0.36}$ & $0.15_{-0.30}^{+0.32}$ & $0.07_{-0.37}^{+0.40}$ \\
\hline$\xi_{a}$ & $0.22_{-0.59}^{+0.78}$ & $0.41_{-0.59}^{+0.59}$ & $0.17_{-0.45}^{+0.83}$ & $0.39_{-0.74}^{+0.69}$ & $0.37_{-0.87}^{+0.81}$ \\
\hline$\Omega_{m 0}$ & $0.30_{-0.15}^{+0.15}$ & $0.321_{-0.098}^{+0.094}$ & $0.312_{-0.097}^{+0.077}$ & $0.195_{-0.044}^{+0.053}$ & $0.229_{-0.052}^{+0.062}$ \\
\hline$\sigma_{8}$ & $0.86_{-0.17}^{+0.19}$ & $0.82_{-0.11}^{+0.12}$ & $0.83_{-0.10}^{+0.12}$ & $0.997_{-0.088}^{+0.083}$ & $0.95_{-0.10}^{+0.09}$ \\
\hline$H_{0}$ & $68.3_{-6.2}^{+6.0}$ & $68.0_{-2.6}^{+2.9}$ & $67.9_{-2.2}^{+2.6}$ & $73.1_{-2.4}^{+2.3}$ & $71.3_{-2.2}^{+2.2}$ \\
\hline$S_{8}$ & $0.846_{-0.068}^{+0.046}$ & $0.845_{-0.039}^{+0.035}$ & $0.840_{-0.039}^{+0.234}$ & $0.800_{-0.047}^{+0.050}$ & $0.826_{-0.042}^{+0.241}$ \\
\hline
\end{tabular}

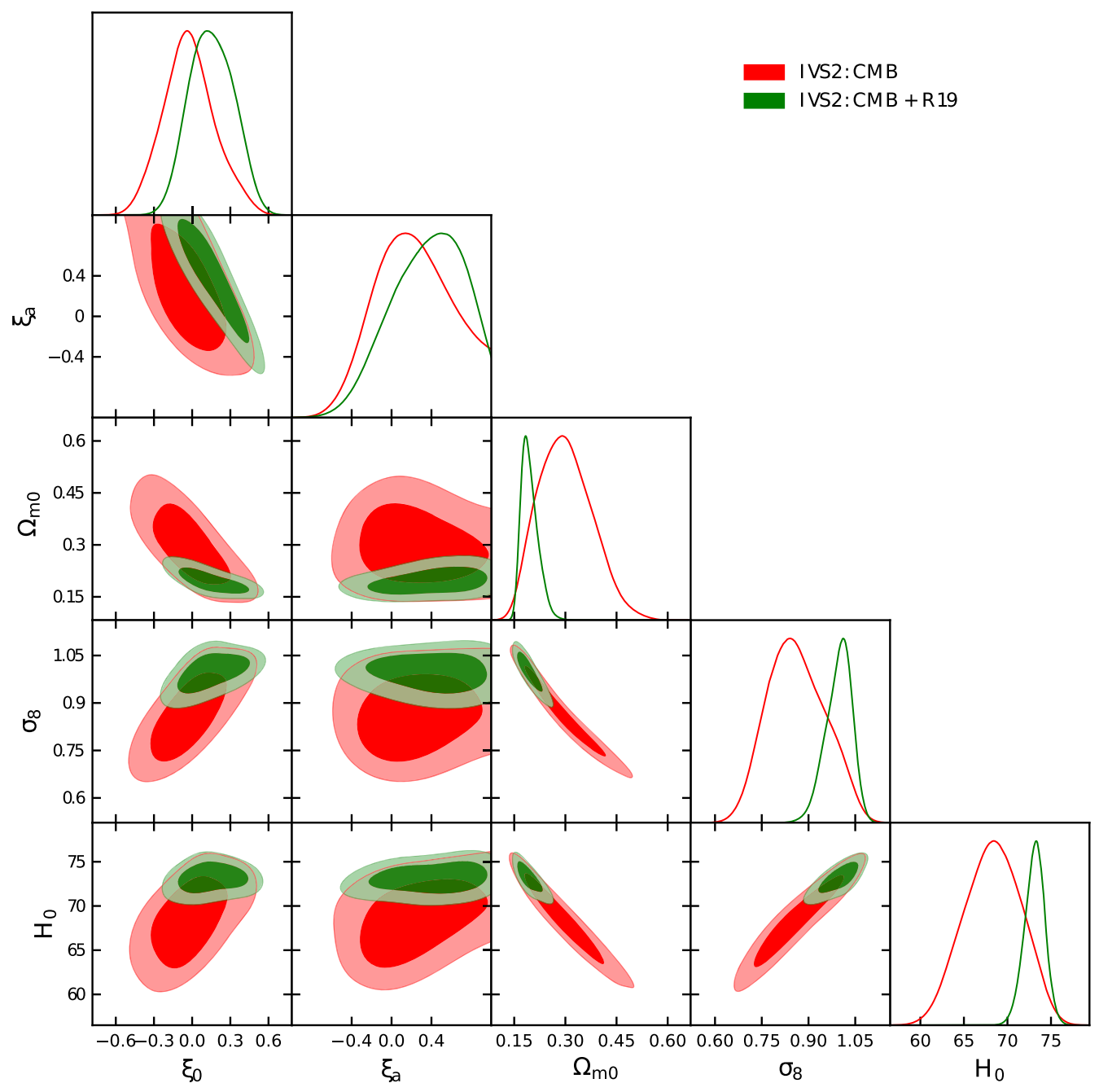

FIG. 4. Two-dimensional contours and one-dimensional marginalized posterior distributions for some key parameters of the IVS2 scenario for the CMB and $\mathrm{CMB}+\mathrm{R} 19$ data sets. 
Finally, when adding the Pantheon data set to $\mathrm{CMB}+$ $\mathrm{BAO}$ (i.e., the combination named $\mathrm{CMB}+\mathrm{BAO}+$ Pantheon in Table II), we note that the estimation of $H_{0}$ shifts down and its error bars are reduced, therefore increasing the tension with R19 measurements.

\section{B. IVS2: $Q=3\left[\xi_{0}+\xi_{a}(1-a)\right] H \frac{\rho_{c} \rho_{x}}{\rho_{c}+\rho_{x}}$}

The summary of the observational constraints on this interaction scenario is shown in Table III, while in Figs. 4 and 5 we depict the one-dimensional marginalized posterior distributions and the two-dimensional contour plots for a number of both independent and derived cosmological parameters, emphasizing their correlations with $\xi_{0}$ and $\xi_{a}$. Note that for CMB-alone data the mean value of $\xi_{0}$ is almost zero, with a very mild preference for negative values, while the mean value of $\xi_{a}$ is found to be positive. When external data sets such as BAO or BAO plus Pantheon are added to $\mathrm{CMB}$ observations, the tendency of $\xi_{0}\left(\xi_{a}\right)$ to take negative (positive) values is enhanced, showing opposite behaviors and a strong negative correlation between them, as we can see from Figs. 4 and 5 regardless of the observations considered in the analysis. Furthermore, from Table III, it is also possible to notice that for all the observational data sets, the values $\left(\xi_{0}, \xi_{a}\right)=$ $(0,0)$ are allowed within $95 \% \mathrm{CL}$, implying that we recover the noninteracting $\Lambda \mathrm{CDM}$ limit. Nevertheless, the dynamical interacting scenario cannot be ruled out, and indeed, based on present observations, no definite conclusion can be made.

Focusing on the estimation of $H_{0}$ for this interaction scenario with CMB data only (see Table III), we notice that it is slightly larger than within the $\Lambda \mathrm{CDM}$ scenario, and its error bars are increased due to the presence of a dynamical coupling. Due to the larger error bars on $H_{0}$, for this model it is also possible to solve the tension with the local measurements of R19 [71]. Combining the CMB data set with a Gaussian prior on $H_{0}$ from R19 therefore provides a possible solution of both the Hubble constant tension and the $S_{8}$ tension with the cosmic shear data.

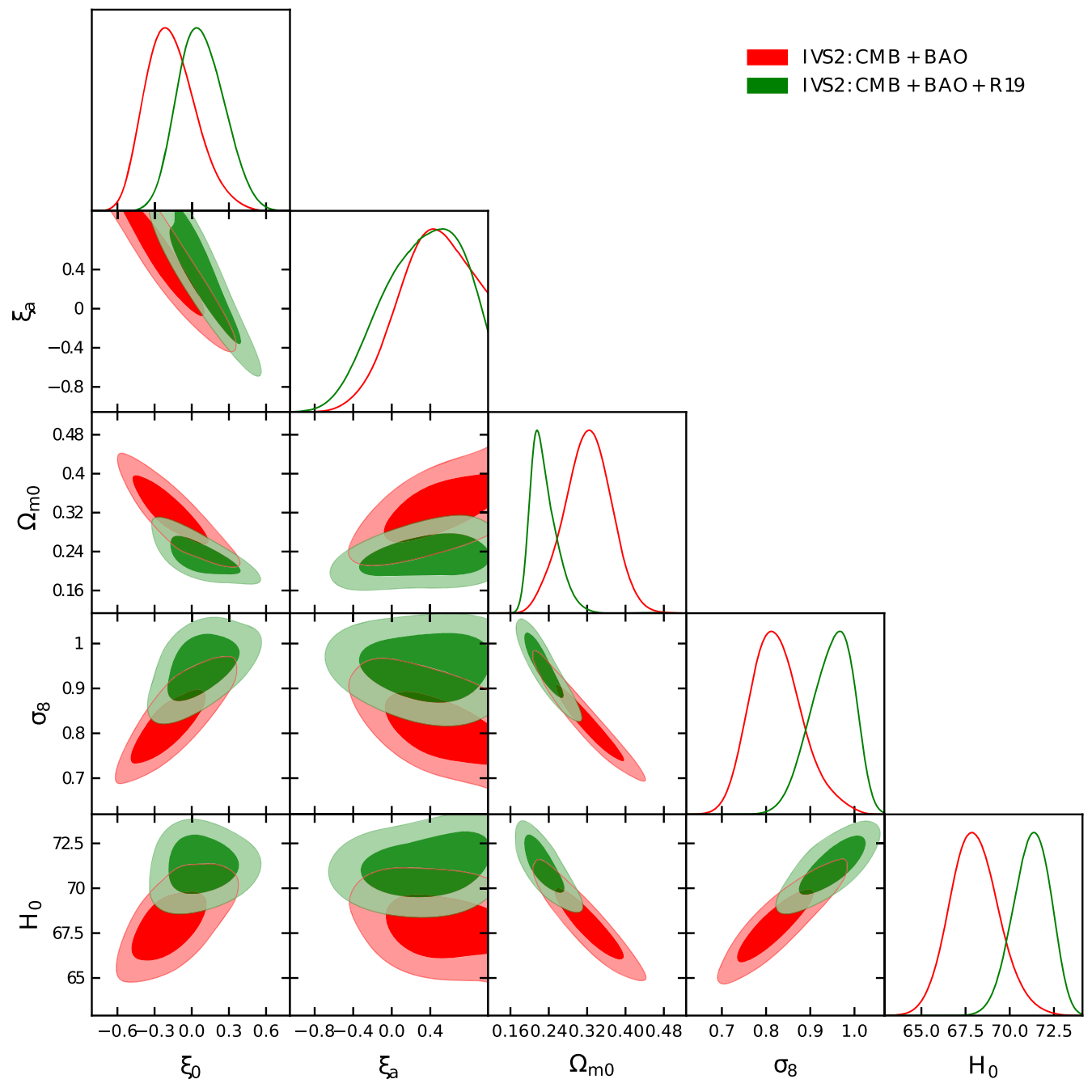

FIG. 5. Two-dimensional contours and one-dimensional marginalized posterior distributions for some key parameters of the IVS2 scenario for the $\mathrm{CMB}+\mathrm{BAO}$ and $\mathrm{CMB}+\mathrm{BAO}+\mathrm{R} 19$ data sets. 

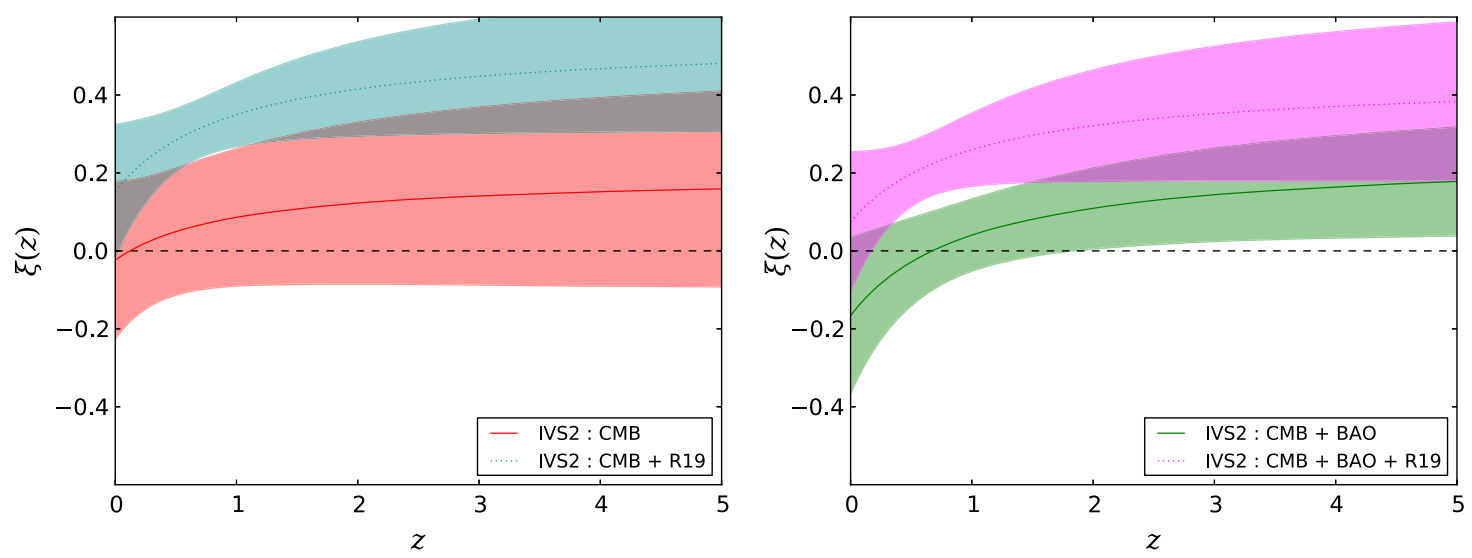

FIG. 6. We show the qualitative evolution of the dynamical coupling parameter $\xi(z)\left(1+z=a^{-1}\right)$ for the IVS2 scenario using various observational data sets. The left panel corresponds to the CMB and CMB + R19 data sets, while the right panel shows for the $\mathrm{CMB}+\mathrm{BAO}$ and $\mathrm{CMB}+\mathrm{BAO}+\mathrm{R} 19$ data sets.

The dynamical evolution of the coupling parameter $\xi(z)$ for this combination of data is shown in Fig. 6; notice that in this case there is also a tendency for $\xi(z)>0$ for $\mathrm{CMB}+\mathrm{R} 19$, and the addition of BAO data is less able to restore $\xi(z)=0$ than within the IVS1 scenario. For $\mathrm{CMB}+\mathrm{BAO}$ or $\mathrm{CMB}+\mathrm{BAO}+$ Pantheon the situation is very similar to the dynamical IVS1 scenario aforementioned; i.e., neither the $H_{0}$ nor the $S_{8}$ tension is alleviated.

Finally, we comment on the results from a Bayesian evidence analysis of the dynamical interacting scenarios explored here. In this framework, a comparison of a cosmological model is performed with respect to a standard and well-motivated cosmological model [84-87]. The $\Lambda \mathrm{CDM}$ provides the ideal choice for such a comparison. We can introduce the so-called Jeffreys scale, which, for different possible values of $\ln B_{i j}$, quantifies the strength of evidence of the reference, the canonical $\Lambda \mathrm{CDM}$ scenario $\left(M_{i}\right)$, with respect to the underlying cosmological model $\left(M_{j}\right)$ [88]. We have, for $0 \leq \ln B_{i j}<1$, weak evidence; for $1 \leq \ln B_{i j}<3$, definite/positive evidence; for $3 \leq \ln B_{i j}<5$, strong evidence; and for $\ln B_{i j} \geq 5$, very strong evidence for the $\Lambda \mathrm{CDM}$ model against the underlying cosmological scenario, here the interacting scenario. Following $[85,86]$

TABLE IV. The table summarizes the values of $\ln B_{i j}$ computed for the $\Lambda$ CDM model with respect to the IVS models.

\begin{tabular}{lcr}
\hline \hline Data set & Model & $\ln B_{i j}$ \\
\hline CMB & IVS1 & 5.9 \\
CMB + BAO & IVS1 & 8.5 \\
CMB + BAO + Pantheon & IVS1 & 4.1 \\
CMB + R19 & IVS1 & 2.8 \\
CMB + BAO + R19 & IVS1 & 9.9 \\
CMB & IVS2 & 5.1 \\
CMB + BAO & IVS2 & 7.7 \\
CMB + BAO + Pantheon & IVS2 & 3.8 \\
CMB + R19 & IVS2 & 3.3 \\
CMB + BAO + R19 & IVS2 & 10.1 \\
\hline \hline
\end{tabular}

we compute the values of $\ln B_{i j}$ for all the observational data sets employed in this work, and we present the results in Table IV. From this table, we learn that the $\Lambda$ CDM model is always preferred over the two IVS models analyzed here. This is not surprising because $\Lambda \mathrm{CDM}$ has six free parameters, while the two IVS models (IVS1 and IVS2) have eight free parameters. This fact eventually favors the base $\Lambda \mathrm{CDM}$ cosmology over the IVS models.

\section{SUMMARY AND CONCLUSIONS}

Noncanonical cosmologies with an interaction between the dark matter and dark energy fluids have been widely investigated in the past several years. From the observational perspective, interacting theories have been found to provide a very promising way to solve the tension between early and late universe cosmological estimates of the Hubble constant. The present work generalizes interacting dark matter-dark energy models by considering a dynamical, redshift-dependent, coupling parameter.

In mostly all of the dark sector interacting theories, characterized by exchange rates $Q=3 H \xi f\left(\rho_{c}, \rho_{x}\right)$ (where $f$ is an analytic function of the arguments $\rho_{c}$ and/or $\rho_{x}$ ), the coupling parameter $\xi$ is assumed to be independent of time (however, see e.g., Ref. [54]). Unless one is interested in minimizing the number of extra parameters in the theory, there exists no underlying symmetry or law in nature which forbids such a dynamical coupling parameter. We have considered a very natural functional form for $\xi=\xi_{0}+$ $\xi_{a}(1-a)$, which we have embedded into two possible interaction models, IVS1 $\left(Q=3\left[\xi_{0}+\xi_{a}(1-a)\right] H \rho_{x}\right)$ and IVS2 $\left(Q=3\left[\xi_{0}+\xi_{a}(1-a)\right] H \frac{\rho_{c} \rho_{x}}{\rho_{c}+\rho_{x}}\right)$.

We find that the interaction parameters $\xi_{0}$ and $\xi_{a}$, governing the dynamical behavior of the coupling $\xi$, are, in almost all cases, perfectly compatible with a noninteracting scenario, showing a strong negative correlation among them. Nevertheless, for the CMB+R19 data combination, we find an indication for $\xi_{a}>0$ at more than $2 \sigma \mathrm{CL}$ 
for IVS1. More importantly, when considering CMB data alone, we find, in general, that $\xi(z)>0$, leading to a smaller value of the present matter density. In order to leave the CMB acoustic peaks location unchanged (which are mostly sensitive to the $\Omega_{m} h^{2}$ combination), a larger value of the Hubble constant $H_{0}$ is required. This in turn implies an optimal scenario to address both the $H_{0}$ and $S_{8}$ tensions between the early and late universe observations.

Even if a Bayesian evidence analysis taking into account all observational data sets shows no particular preference for these interacting dark matter-dark energy models, a dynamical character in the interaction functions is still allowed by observations and can solve some pending issues related to high- and low-redshift cosmological tensions.

\section{ACKNOWLEDGMENTS}

The authors thank the referees for their important comments and suggestions aiming to improve the manuscript.
W. Y. acknowledges the support from the National Natural Science Foundation of China under Grants No. 11705079 and No. 11647153. O.M. is supported by the Spanish Grants No. FPA2017-85985-P and No. SEV-2014-0398 of the MINECO and the European Union's Horizon 2020 research and innovation program under the Grant Agreements No. 690575 and No. 674896. S. P. gratefully acknowledges several fruitful discussions with Professor J. D. Barrow on this topic. The research of S. P. has been supported by the Mathematical Research Impact-Centric Support Scheme (MATRICS), File No. MTR/2018/ 000940, given by the Science and Engineering Research Board (SERB), Government of India, and also by the Faculty Research and Professional Development Fund (FRPDF) Scheme of Presidency University, Kolkata, India. E. D. V. acknowledges support from the European Research Council in the form of Consolidator Grant No. 681431.
[1] N. Aghanim et al. (Planck Collaboration), Planck 2018 results. VI. Cosmological parameters, arXiv:1807.06209.

[2] N. Suzuki et al., The Hubble space telescope cluster supernova survey: V. Improving the dark energy constraints above $z>1$ and building an early-type-hosted supernova sample, Astrophys. J. 746, 85 (2012).

[3] M. Crocce et al. (DES Collaboration), Galaxy clustering, photometric redshifts and diagnosis of systematics in the DES Science Verification data, Mon. Not. R. Astron. Soc. 455, 4301 (2016).

[4] S. Alam et al. (SDSS-III Collaboration), The eleventh and twelfth data releases of the Sloan digital sky survey: Final data from SDSS-III, Astrophys. J. Suppl. Ser. 219, 12 (2015).

[5] G. Hinshaw et al. (WMAP Collaboration), Nine-year Wilkinson microwave anisotropy probe (WMAP) observations: Cosmological parameter results, Astrophys. J. Suppl. Ser. 208, 19 (2013).

[6] P. A. R. Ade et al. (Planck Collaboration), Planck 2015 results. XIII. Cosmological parameters, Astron. Astrophys. 594, A13 (2016).

[7] S. M. Carroll, Quintessence and the Rest of the World: Suppressing Long-Range Interactions, Phys. Rev. Lett. 81, 3067 (1998).

[8] C. Wetterich, The Cosmon model for an asymptotically vanishing time dependent cosmological "constant," Astron. Astrophys. 301, 321 (1995).

[9] L. Amendola, Coupled quintessence, Phys. Rev. D 62, 043511 (2000).

[10] L. Amendola and C. Quercellini, Tracking and coupled dark energy as seen by WMAP, Phys. Rev. D 68, 023514 (2003).

[11] D. Pavón and W. Zimdahl, Holographic dark energy and cosmic coincidence, Phys. Lett. B 628, 206 (2005).
[12] S. del Campo, R. Herrera, and D. Pavón, Toward a solution of the coincidence problem, Phys. Rev. D 78, 021302 (2008).

[13] S. del Campo, R. Herrera, and D. Pavón, Interacting models may be key to solve the cosmic coincidence problem, J. Cosmol. Astropart. Phys. 01 (2009) 020.

[14] S. Das, P. S. Corasaniti, and J. Khoury, Super-acceleration as signature of dark sector interaction, Phys. Rev. D 73, 083509 (2006).

[15] H. M. Sadjadi and M. Honardoost, Thermodynamics second law and $\omega=-1$ crossing(s) in interacting holographic dark energy model, Phys. Lett. B 647, 231 (2007).

[16] S. Pan and S. Chakraborty, A cosmographic analysis of holographic dark energy models, Int. J. Mod. Phys. D 23, 1450092 (2014).

[17] A. P. Billyard and A. A. Coley, Interactions in scalar field cosmology, Phys. Rev. D 61, 083503 (2000).

[18] J. D. Barrow and T. Clifton, Cosmologies with energy exchange, Phys. Rev. D 73, 103520 (2006).

[19] L. Amendola, G. Camargo Campos, and R. Rosenfeld, Consequences of dark matter-dark energy interaction on cosmological parameters derived from SNIa data, Phys. Rev. D 75, 083506 (2007).

[20] J. H. He and B. Wang, Effects of the interaction between dark energy and dark matter on cosmological parameters, J. Cosmol. Astropart. Phys. 06 (2008) 010.

[21] J. Väliviita, E. Majerotto, and R. Maartens, Instability in interacting dark energy and dark matter fluids, J. Cosmol. Astropart. Phys. 07 (2008) 020.

[22] M. B. Gavela, D. Hernandez, L. Lopez Honorez, O. Mena, and S. Rigolin, Dark coupling, J. Cosmol. Astropart. Phys. 07 (2009) 034. 
[23] E. Majerotto, J. Valiviita, and R. Maartens, Adiabatic initial conditions for perturbations in interacting dark energy models, Mon. Not. R. Astron. Soc. 402, 2344 (2010).

[24] M. B. Gavela, L. Lopez Honorez, O. Mena, and S. Rigolin, Dark coupling and gauge invariance, J. Cosmol. Astropart. Phys. 11 (2010) 044.

[25] T. Clemson, K. Koyama, G. B. Zhao, R. Maartens, and J. Valiviita, Interacting dark energy-Constraints and degeneracies, Phys. Rev. D 85, 043007 (2012).

[26] S. Pan, S. Bhattacharya, and S. Chakraborty, An analytic model for interacting dark energy and its observational constraints, Mon. Not. R. Astron. Soc. 452, 3038 (2015).

[27] S. Pan and S. Chakraborty, Will there be again a transition from acceleration to deceleration in course of the dark energy evolution of the Universe?, Eur. Phys. J. C 73, 2575 (2013).

[28] W. Yang and L. Xu, Testing coupled dark energy with large scale structure observation, J. Cosmol. Astropart. Phys. 08 (2014) 034.

[29] W. Yang and L. Xu, Cosmological constraints on interacting dark energy with redshift-space distortion after Planck data, Phys. Rev. D 89, 083517 (2014).

[30] S. Pan and G. S. Sharov, A model with interaction of dark components and recent observational data, Mon. Not. R. Astron. Soc. 472, 4736 (2017).

[31] A. Mukherjee and N. Banerjee, In search of the dark matter dark energy interaction: A kinematic approach, Classical Quantum Gravity 34, 035016 (2017).

[32] G. S. Sharov, S. Bhattacharya, S. Pan, R. C. Nunes, and S. Chakraborty, A new interacting two fluid model and its consequences, Mon. Not. R. Astron. Soc. 466, 3497 (2017).

[33] W. Yang, N. Banerjee, and S. Pan, Constraining a dark matter and dark energy interaction scenario with a dynamical equation of state, Phys. Rev. D 95, 123527 (2017).

[34] W. Yang, S. Pan, and D. F. Mota, Novel approach toward the large-scale stable interacting dark-energy models and their astronomical bounds, Phys. Rev. D 96, 123508 (2017).

[35] S. Pan, A. Mukherjee, and N. Banerjee, Astronomical bounds on a cosmological model allowing a general interaction in the dark sector, Mon. Not. R. Astron. Soc. 477, 1189 (2018).

[36] W. Yang, S. Pan, and A. Paliathanasis, Cosmological constraints on an exponential interaction in the dark sector, Mon. Not. R. Astron. Soc. 482, 1007 (2019).

[37] W. Yang, S. Pan, L. Xu, and D. F. Mota, Effects of anisotropic stress in interacting dark matter dark energy scenarios, Mon. Not. R. Astron. Soc. 482, 1858 (2019).

[38] W. Yang, S. Pan, R. Herrera, and S. Chakraborty, Largescale (in)stability analysis of an exactly solved coupled dark-energy model, Phys. Rev. D 98, 043517 (2018).

[39] M. Martinelli, N. B. Hogg, S. Peirone, M. Bruni, and D. Wands, Constraints on the interacting vacuum-geodesic CDM scenario, Mon. Not. R. Astron. Soc. 488, 3423 (2019).

[40] A. Paliathanasis, S. Pan, and W. Yang, Dynamics of nonlinear interacting dark energy models, arXiv:1903.02370.

[41] S. Pan, W. Yang, C. Singha, and E. N. Saridakis, Observational constraints on sign-changeable interaction models and alleviation of the $H_{0}$ tension, arXiv:1903.10969.
[42] W. Yang, S. Pan, E. Di Valentino, B. Wang, and A. Wang, Forecasting interacting vacuum-energy models using gravitational waves, arXiv:1904.11980.

[43] W. Yang, S. Vagnozzi, E. Di Valentino, R. C. Nunes, S. Pan, and D. F. Mota, Listening to the sound of dark sector interactions with gravitational wave standard sirens, J. Cosmol. Astropart. Phys. 07 (2019) 037.

[44] S. Kumar and R. C. Nunes, Echo of interactions in the dark sector, Phys. Rev. D 96, 103511 (2017).

[45] E. Di Valentino, A. Melchiorri, and O. Mena, Can interacting dark energy solve the $H_{0}$ tension? Phys. Rev. D 96, 043503 (2017).

[46] W. Yang, S. Pan, E. Di Valentino, R. C. Nunes, S. Vagnozzi, and D.F. Mota, Tale of stable interacting dark energy, observational signatures, and the $H_{0}$ tension, J. Cosmol. Astropart. Phys. 09 (2018) 019.

[47] W. Yang, A. Mukherjee, E. Di Valentino, and S. Pan, Interacting dark energy with time varying equation of state and the $H_{0}$ tension, Phys. Rev. D 98, 123527 (2018).

[48] V. Salvatelli, N. Said, M. Bruni, A. Melchiorri, and D. Wands, Indications of a Late-Time Interaction in the Dark Sector, Phys. Rev. Lett. 113, 181301 (2014).

[49] R. C. Nunes, S. Pan, and E. N. Saridakis, New constraints on interacting dark energy from cosmic chronometers, Phys. Rev. D 94, 023508 (2016).

[50] S. Kumar and R. C. Nunes, Probing the interaction between dark matter and dark energy in the presence of massive neutrinos, Phys. Rev. D 94, 123511 (2016).

[51] C. van de Bruck, J. Mifsud, and J. Morrice, Testing coupled dark energy models with their cosmological background evolution, Phys. Rev. D 95, 043513 (2017).

[52] W. Yang, H. Li, Y. Wu, and J. Lu, Cosmological constraints on coupled dark energy, J. Cosmol. Astropart. Phys. 10 (2016) 007.

[53] W. Yang, S. Pan, and J. D. Barrow, Large-scale stability and astronomical constraints for coupled dark-energy models, Phys. Rev. D 97, 043529 (2018).

[54] Y. Wang and G. B. Zhao, Constraining the dark mattervacuum energy interaction using the EDGES 21-cm absorption signal, Astrophys. J. 869, 26 (2018).

[55] Y.H. Li and X. Zhang, Running coupling: Does the coupling between dark energy and dark matter change sign during the cosmological evolution?, Eur. Phys. J. C 71, 1700 (2011).

[56] J. J. Guo, J. F. Zhang, Y. H. Li, D. Z. He, and X. Zhang, Probing the sign-changeable interaction between dark energy and dark matter with current observations, Sci. China Phys. Mech. Astron. 61, 030011 (2018).

[57] V. F. Mukhanov, H. A. Feldman, and R. H. Brandenberger, Theory of cosmological perturbations, Phys. Rep. 215, 203 (1992).

[58] C. P. Ma and E. Bertschinger, Cosmological perturbation theory in the synchronous and conformal Newtonian gauges, Astrophys. J. 455, 7 (1995).

[59] K. A. Malik and D. Wands, Cosmological perturbations, Phys. Rep. 475, 1 (2009).

[60] Y. Wang, D. Wands, G. B. Zhao, and L. Xu, Post-Planck constraints on interacting vacuum energy, Phys. Rev. D 90, 023502 (2014). 
[61] A. Shafieloo, D. K. Hazra, V. Sahni, and A. A. Starobinsky, Metastable dark energy with radioactive-like decay, Mon. Not. R. Astron. Soc. 473, 2760 (2018).

[62] R. G. Landim and E. Abdalla, Metastable dark energy, Phys. Lett. B 764, 271 (2017).

[63] M. Szydłowski, A. Stachowski, and K. Urbanowski, Quantum mechanical look at the radioactive-like decay of metastable dark energy, Eur. Phys. J. C 77, 902 (2017).

[64] X. L. Li, A. Shafieloo, V. Sahni, and A. A. Starobinsky, Revisiting metastable dark energy and tensions in the estimation of cosmological parameters, arXiv:1904.03790.

[65] R. Adam et al. (Planck Collaboration), Planck 2015 results. I. Overview of products and scientific results, Astron. Astrophys. 594, A1 (2016).

[66] N. Aghanim et al. (Planck Collaboration), Planck 2015 results. XI. CMB power spectra, likelihoods, and robustness of parameters, Astron. Astrophys. 594, A11 (2016).

[67] F. Beutler, C. Blake, M. Colless, D. H. Jones, L. StaveleySmith, L. Campbell, Q. Parker, W. Saunders, and F. Watson, The 6dF Galaxy survey: Baryon acoustic oscillations and the local hubble constant, Mon. Not. R. Astron. Soc. 416, 3017 (2011).

[68] A. J. Ross, L. Samushia, C. Howlett, W. J. Percival, A. Burden, and M. Manera, The clustering of the SDSS DR7 main Galaxy sample-I. A 4 per cent distance measure at $z=0.15$, Mon. Not. R. Astron. Soc. 449, 835 (2015).

[69] H. Gil-Marín et al., The clustering of galaxies in the SDSSIII Baryon Oscillation Spectroscopic Survey: BAO measurement from the LOS-dependent power spectrum of DR12 BOSS galaxies, Mon. Not. R. Astron. Soc. 460, 4210 (2016).

[70] D. M. Scolnic et al., The complete light-curve sample of spectroscopically confirmed SNe Ia from Pan-STARRS1 and cosmological constraints from the combined pantheon sample, Astrophys. J. 859, 101 (2018).

[71] A. G. Riess, S. Casertano, W. Yuan, L. M. Macri, and D. Scolnic, Large magellanic cloud Cepheid standards provide a $1 \%$ foundation for the determination of the Hubble constant and stronger evidence for physics beyond

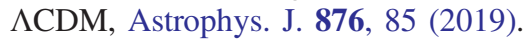

[72] A. Lewis and S. Bridle, Cosmological parameters from CMB and other data: A Monte Carlo approach, Phys. Rev. D 66, 103511 (2002).

[73] A. Lewis, A. Challinor, and A. Lasenby, Efficient computation of CMB anisotropies in closed FRW models, Astrophys. J. 538, 473 (2000).
[74] E. Di Valentino and S. Bridle, Exploring the tension between current cosmic microwave background and cosmic shear data, Symmetry 10, 585 (2018).

[75] K. Kuijken et al., Gravitational lensing analysis of the kilo degree survey, Mon. Not. R. Astron. Soc. 454, 3500 (2015).

[76] H. Hildebrandt et al., KiDS-450: Cosmological parameter constraints from tomographic weak gravitational lensing, Mon. Not. R. Astron. Soc. 465, 1454 (2017).

[77] I. F. Conti, R. Herbonnet, H. Hoekstra, J. Merten, L. Miller, and M. Viola, Calibration of weak-lensing shear in the KiloDegree Survey, Mon. Not. R. Astron. Soc. 467, 1627 (2017).

[78] T. M. C. Abbott et al. (DES Collaboration), Dark energy survey year 1 results: Cosmological constraints from galaxy clustering and weak lensing, Phys. Rev. D 98, 043526 (2018).

[79] M. A. Troxel et al. (DES Collaboration), Dark energy survey year 1 results: Cosmological constraints from cosmic shear, Phys. Rev. D 98, 043528 (2018).

[80] C. Heymans et al., CFHTLenS: The Canada-France-Hawaii telescope lensing survey, Mon. Not. R. Astron. Soc. 427, 146 (2012).

[81] T. Erben et al., CFHTLenS: The Canada-France-Hawaii Telescope Lensing Survey-Imaging data and catalogue products, Mon. Not. R. Astron. Soc. 433, 2545 (2013).

[82] S. Joudaki et al., CFHTLenS revisited: Assessing concordance with Planck including astrophysical systematics, Mon. Not. R. Astron. Soc. 465, 2033 (2017).

[83] X. Zhang and Q. G. Huang, Constraints on $H_{0}$ from WMAP and BAO measurements, Commun. Theor. Phys. 71, 826 (2019).

[84] W. Yang, S. Pan, E. Di Valentino, E. N. Saridakis, and S. Chakraborty, Observational constraints on one-parameter dynamical dark-energy parametrizations and the $H_{0}$ tension, Phys. Rev. D 99, 043543 (2019).

[85] A. Heavens, Y. Fantaye, A. Mootoovaloo, H. Eggers, Z. Hosenie, S. Kroon, and E. Sellentin, Marginal likelihoods from Monte Carlo Markov chains, arXiv:1704.03472.

[86] A. Heavens, Y. Fantaye, E. Sellentin, H. Eggers, Z. Hosenie, S. Kroon, and A. Mootoovaloo, No Evidence for Extensions to the Standard Cosmological Model, Phys. Rev. Lett. 119, 101301 (2017).

[87] S. Pan, E. N. Saridakis, and W. Yang, Observational constraints on oscillating dark-energy parametrizations, Phys. Rev. D 98, 063510 (2018).

[88] R. E. Kass and A. E. Raftery, Bayes factors, J. Am. Stat. Assoc. 90, 773 (1995). 